Research Article

\title{
Nonlinear Analysis Method of High-Strength Steel Based on Local Buckling Fiber Hinge
}

\author{
Yuhua Wang (iD) and Hengchao Zheng (D) \\ School of Electricity and Engineering, Nanjing Institute of Railway Technology, Nanjing, Jiangsu 210031, China \\ Correspondence should be addressed to Hengchao Zheng; zhenghengchao@njrts.edu.cn
}

Received 24 February 2021; Revised 30 March 2021; Accepted 2 April 2021; Published 24 April 2021

Academic Editor: Yi-Zhang Jiang

Copyright (C) 2021 Yuhua Wang and Hengchao Zheng. This is an open access article distributed under the Creative Commons Attribution License, which permits unrestricted use, distribution, and reproduction in any medium, provided the original work is properly cited.

\begin{abstract}
Based on the analysis and summary of the research and application status of domestic and foreign high-strength steel local buckling fiber hinged rod stability and ordinary steel local buckling fiber hinged rod stability control, this paper proposes a fiber hinged rod suitable for spatial structures subject to local buckling. The new type is high-strength steel composite local buckling fiber hinged pressure rod. The influence of related parameters in the dislocation string model and the dislocation couple model on the ultrasonic nonlinear parameters is deeply analyzed. And, from the perspective of contact nonlinear acoustics, the mechanism of the ultrasonic nonlinear response of the crack is analyzed, and the finite element software ABAQUS is used to simulate it. The relationship between the nonlinear parameter and the internal crack shape of the material is simulated and analyzed, which proves the nonlinearity. A series of nonlinear ultrasonic testing was performed on three groups of FV520B high-strength steel fatigue specimens using a nonlinear testing system. Analyzing the results, it is found that the material has a good ultrasonic nonlinear cumulative effect, and the microcracks have a greater impact on the ultrasonic nonlinear response. The $\beta$-N curves under three sets of fatigue tests are obtained. The results show that the nonlinear parameters are very sensitive to the fatigue damage of FV520B high-strength steel, and the ultrasonic nonlinear parameters generally increase with the increase in the number of fatigue cycles.
\end{abstract}

\section{Introduction}

High-strength steel refers to steel with a standard value of yield strength between 460 and $690 \mathrm{MPa}$. With the development of new steel production processes, such as microalloying technology and thermomechanical treatment technology, highstrength steel, especially compared to earlier high-strength steels, has a higher cleanliness; $\mathrm{Nb}, \mathrm{V}$, and Ti are used as the elements. The representative microalloying replaces the traditional carbon element strengthening method, which not only improves the yield strength of steel, but also improves its plasticity and toughness [1]. The new high-strength steel developed by this new process has the characteristics of high strength, good toughness, and good processing and weldability and has been applied in many practical projects at home and abroad $[2,3]$.
For high-strength steel welded I-shaped section members, in order to make full use of the advantages of high strength, the section design can be more developed, but this can easily exceed the current specification limits, especially the height-to-thickness ratio of the web [4]. Under the load of the axial compression member with the web height-tothickness ratio exceeding the limit, the web buckles first, but the bearing capacity of the entire member does not reach the maximum, and there is still a lot of room for ascent $[5,6]$. Therefore, it is necessary to appropriately relax the heightto-thickness ratio limit of the web, so that it can give full play to its own strength advantages, and also improve the economics of welding I-shaped cross section members $[7,8]$. However, there are relatively few studies on high-strength steels with a yield strength of $690 \mathrm{MPa}$, and there are even fewer design codes applicable to it. The existing 
specifications for high-strength steels simply apply design formulas suitable for ordinary-strength steels, which greatly restricts the promotion and application of high-strength steel structures [9].

This paper proposes a simplified calculation model of the composite local buckling fiber hinged compression rod (using the BEAM188 element node coupling method), and the correctness of the simplified calculation model is verified by comparison and analysis with solid elements. In the initial defect, the residual stress will have an unfavorable effect on the stable bearing capacity. In this paper, the analysis of the local buckling fiber hinged compression rod of high-strength steel with different slenderness ratios shows that the welding residual stress has little effect on the stable bearing capacity (when the residual stress is not considered, the maximum difference of the stable bearing capacity is only $3.02 \%)$. In this paper, the fabricated FV520B high-strength steel fatigue specimen was tested by a nonlinear ultrasonic testing system. Through the data collection and processing, the fundamental wave and the second harmonic amplitude were extracted. The relationship between the ultrasonic nonlinear parameters and the ultrasonic propagation distance in the material was studied. The ultrasonic nonlinear response was detected in the original plate specimen without fatigue damage, indicating the inherent nonlinear characteristics of the material itself. As the propagation distance increases, the ultrasonic nonlinear coefficient increases approximately linearly, indicating that the nonlinear response has a cumulative effect. Testing the notched samples that cycle for a certain number of cycles also found the cumulative effect of the ultrasonic nonlinear response. The $\beta-\mathrm{N}$ curves of FV520B material under three stress levels are obtained. The results show that the nonlinear parameters are very sensitive to the early fatigue damage of FV520B high-strength steel. The nonlinear parameters show an increasing trend with the increase of the number of fatigue cycles. The relationship between the ultrasonic nonlinear parameters of FV520B high-strength steel and the fatigue cycle cycles can be used to characterize the early fatigue damage degree of the material and reflect its fatigue life.

\section{Related Work}

At present, there are still few domestic studies on the overall stability and local stability of high-strength steel beams [10]. Relevant scholars have studied the stability of high-strength steel thin-walled box-section beams, mainly analyzing the effects of the slenderness ratio, flange width-to-thickness ratio, and section side-length ratio of the box beam on its overall stability [11]. The steel studied is $6 \mathrm{~mm}$ thick, $18 \mathrm{Mn} 2 \mathrm{Cr}$ Mo BA high-strength steel with a yield strength of $745 \mathrm{MPa}$. This type of steel is generally used in bridge engineering. Relevant scholars pointed out that the slenderness ratio has little effect on the ultimate load of a box-shaped beam in a purely bending state [12]. After changing the slenderness ratio of the beam, the ultimate load changes within $5 \%$. The flange width of the beam is relatively small. After the load-displacement curve appears at the highest point, the curve slowly and steadily declines; when the flange width-to-thickness ratio of the beam is increased, the flange or web has already experienced local instability after the load is added to the ultimate load [13]. The elastic section modulus of the beam decreases, so the slope of the descending section after the highest point of the load-displacement curve increases significantly. It can be seen that the width-to-thickness ratio of the flange affects the overall stability of the beam to a greater extent. When the crosssectional side length ratio of the beam increases from 30 to 60 , the web height-to-thickness ratio continues to increase, and local instability is prone to occur in the compression zone of the web, which leads to an acceleration of the overall stability of the ultimate load drop [14].

The research on the local-total related buckling of boxshaped cross section members has a long history. Domestic and foreign researches mainly focus on the local-total related buckling of ordinary steel members. The research on the local-total related buckling of high-strength steel members mainly focuses on the axis. Relevant scholars have used numerical analysis methods to study the relative buckling ultimate bearing capacity of ordinary steel members with thin-walled box-shaped sections under axial compression with hinged ends [15]. Research has found that when the ultimate bearing capacity of the component is reached, the bearing capacity of the component composed of weak panels will decrease sharply [16]. The researchers used the test results of four axial compression members with a nominal yield strength of $390 \mathrm{MPa}$ to compare with the finite element model [17]. The research shows that residual stress and local overall geometric defects have a great influence on the ultimate bearing capacity of members. Researchers have proposed a formula for calculating the local-to-whole relative buckling ultimate bearing capacity of axial compression members with large width-to-thickness ratio based on the effective width method [18]. Relevant scholars have conducted experimental research on three S355 steel welded thin-walled box-shaped eccentric components, established a finite element model considering the effects of initial geometric defects and residual stress, and compared the experimental results, and they found that the two are in good agreement. Finally, the verified finite element model is used to study the influence of initial defects on the ultimate bearing capacity of the component [19].

Relevant scholars have, respectively, measured and analyzed the overall geometric initial defects of I-shaped section members with nominal yield strengths of $460 \mathrm{MPa}$ and $960 \mathrm{MPa}$ [20]. The results show that the measured amplitude of the overall geometric initial defects of most high-strength steels is less than $1 / 1000$. The researchers measured and analyzed the overall geometric initial defects of the I-shaped section members with nominal yield strengths of $460 \mathrm{MPa}, 550 \mathrm{MPa}$, and $690 \mathrm{MPa}$, and the results showed that the maximum amplitudes were 1/541, 1/339, and 1/702 [21]. Relevant scholars have studied the effect of geometric initial defects on the stability of ordinary steel and high-strength steel [22]. The results show that, compared with ordinary steel members, geometric initial defects have less effect on the stability coefficient of high-strength steel members; compared with the ultimate bearing capacity, the local buckling capacity is more 
sensitive to local geometric initial imperfections. Researchers have conducted experimental research and numerical analysis on the overall stability and local stability of high-strength steel axial compression members and have accumulated a wealth of experimental data and important research results [23]. In particular, the residual stress distribution of high-strength steel welded components is measured by the cutting method, and the residual stress distribution model suitable for different steel strength grades is summarized, which provides reliable residual stress treatment for the future finite element analysis of highstrength steel welded components method. Relevant scholars use ANSYS finite element software to simulate the axial compression components of hot-rolled high-strength equilateral angle steel. Studies have shown that the initial defects have a smaller effect on the local stability bearing capacity of high-strength hot-rolled equilateral angle steel axial compression members than ordinary steel axial compression members. The researchers analyzed and studied the ultimate bearing capacity of Q345, Q390, and Q420 steel welded boxsection axial compression members whose aspect ratio exceeds the limit. The calculation results are compared with the direct strength method and the effective yield strength method. The study shows that the stability coefficient should be checked according to the type a section in the 03 specification; the direct strength method and the finite element calculation result are in good agreement, while the effective yield strength method is slightly conservative.

\section{Steel Tube Buckling Theory and Finite Element Simulation Method}

3.1. The Basic Theory of Axial Buckling. According to the deformation state of the member during buckling, it can be divided into global buckling, local buckling, and global-local related buckling. When the component is only buckled as a whole, its parts will not be severely deformed, and only the whole body will undergo a huge change in configuration before and after buckling; local buckling means that, after buckling of the component, only the local elements will deform significantly; related buckling is a coupling of global buckling and local buckling; that is, when buckling occurs, both the overall and local elements of the member undergo a large configuration change. Due to the inevitable existence of local and overall defects, related buckling is very common in actual engineering and should be paid attention to.

As shown in Figure 1, a cylindrical shell subjected to axial pressure can only maintain a balanced state under conditions far below the critical buckling load after local buckling. Its load-displacement curve is shown in Figure 1. $O A B$ ' belongs to unstable bifurcation instability, and this form of buckling is also called limited interference buckling. Under minimal external interference, the cylindrical shell may jump from the stable equilibrium state before buckling to the nonadjacent equilibrium state before reaching the critical load. The path is shown in the curve OA'CB in Figure 1. It should be noted that geometric defects have a great influence on this type of member, making its actual ultimate bearing capacity far less than the elastic critical buckling load Pcr.
3.2. Calculation Method of Elastic Buckling Problem. The calculation of structural buckling is a complex and tedious process, which involves a series of geometric parameters and initial conditions of the component, such as the structural system, the length of the component, the connection condition, the section form, the size of the initial defect, and the residual caused by the processing and manufacturing of the component.

The static balance method is one of the most important methods to solve the elastic stability limit load of the structure. According to the force situation of the structure with small deformation after the buckling of the member, the buckling load is solved by the mechanical balance equation. Usually more than one buckling load is obtained according to this method, and the minimum value should be taken as the true buckling load of the member. The balance method fully satisfies the mechanical equilibrium state of the component after buckling, so its calculation result is an accurate value. However, it is difficult to find that the balance method is only suitable for simple mechanical models and single boundary conditions. It is generally difficult to obtain the buckling load of components with various forms of midsections and complex forces and boundary conditions using the balance method.

The emergence of the energy method can solve the problem of elastic stability with complex force conditions or structures with diverse forms and compositions. When the structure is subjected to conservative forces, the total potential energy function can be established according to its deformed state. At this time, the total potential energy should include the potential energy caused by the external load of the component and the strain energy caused by the deformation of the internal structure. According to the principle of standing value of potential energy, the potential energy has a standing value when the component is in equilibrium, so the buckling load solution is carried out in two steps. Firstly, the balance equation is obtained by taking the first-order variation of the total potential energy to the displacement, and then the bifurcation buckling load is obtained by solving the balance equation. Different from the balance method, the buckling load obtained by the energy method is generally only an approximate solution, but if the structure deformation form and displacement function can be determined in advance, the exact solution can be obtained. It is worth mentioning that the energy method can judge the stability of bifurcation buckling according to the positive and negative values of the second-order variation of the total potential energy; that is, when the second-order variation is negative, the structure is unstable bifurcation buckling.

\section{Theoretical Analysis of the Stability of Axially Compressed Cylindrical Shells}

4.1. Theoretical Analysis of Linear Small Deflection. For the linear small deflection theory, analysis can be made from two aspects: axisymmetric and nonaxisymmetric. When the axial compression load reaches the critical buckling stress, the steel pipe member may undergo symmetrical deformation and failure of the steel pipe wall "convex" or "concave." 
According to the theory of linear small deflection buckling, the buckling equilibrium differential equation of a cylindrical shell bearing uniform axial pressure is

$$
w G \nabla^{8}+\frac{t E \partial^{4} w}{r \partial x^{4}}+N_{x} \nabla^{4} \frac{\partial^{2} w}{\partial x^{2}}=0 .
$$

$W$ is the displacement perpendicular to the shell surface, $G$ is the bending stiffness of the unit width bridge, $r$ and $t$ are the radius and thickness of the shell, and $N_{x}$ is the pressure on the unit arc length.

We take the buckling deformation displacement function as

$$
w=w_{1} \sin \frac{\pi}{l_{x}} x \cdot \cos \frac{\pi}{l_{y}} y .
$$

In the formula, $l_{x}$ and $l_{y}$ are the buckling half-wave lengths in the $x$ and $y$ directions, respectively. After finishing, the critical buckling stress can be obtained:

$$
N_{x}=G \pi^{2} \psi-\frac{t E}{r^{2} \pi^{2} \psi} .
$$

Among them,

$$
\psi=\frac{1}{l_{x}^{2}} \cdot\left(1-\frac{l_{x}^{2}}{l_{y}^{2}}\right)^{2} .
$$

The minimum value of $\mathrm{Nx}$ is

$$
N_{x}=\frac{t \cdot E}{r \cdot \sqrt{3\left(v^{2}-1\right)}}
$$

Taking steel Poisson's ratio 0.3, then

$$
N_{x}=0.582 \cdot \frac{t E}{r} \text {. }
$$

In actual engineering problems, there are unavoidable geometric initial defects in the components, and the shell is more sensitive to defects. Therefore, the ultimate stress of a cylindrical shell in experiments or actual engineering is always much lower than the elastic critical buckling stress of a cylindrical short column under axial compression. In order to explore the ultimate bearing capacity of cylindrical shells with geometric initial imperfections, it is necessary to adopt the buckling analysis theory considering the nonlinear term.

4.2. Theoretical Analysis of Nonlinear Large Deflection. The nonlinear large deflection buckling theory is proposed on the basis of the linear small deflection theory. There is still another postbuckling equilibrium configuration far below the linear critical load value, and this postbuckling configuration is closer to the real experimental phenomenon. In this study, only the calculation formula for the elastic critical buckling stress of a short cylindrical tube under axial compression obtained by the small deflection theory is considered, so the detailed calculation method of the large deflection buckling theory will not be repeated.

\section{Main Factors Affecting Local Buckling of Cylindrical Shells under Axial Compression}

5.1. Influence of Boundary Conditions. When the two ends of the member are unconstrained and can move freely in the circumferential direction, the critical buckling load will be significantly reduced. However, in actual engineering structures, the ends of circular pipe members usually have constrained members such as gusset plates and end bearing plates. The end can be freely deformed rarely. Therefore, for the local buckling of the axially compressed cylindrical short column, the influence of the component due to the boundary conditions can be ignored.

5.2. Influence of Residual Stress. Welding is a physical process of local heating and then cooling of component materials. Due to the uneven temperature field of the heating area and surrounding materials, the welding area will deform unevenly during the cooling process and cause various welding residual stresses. Therefore, the residual stress is an unavoidable unfavorable factor for welded pipe members. In-depth tests and numerical analysis can be done on the residual stress distribution pattern of welded circular pipe components and its influence on the ultimate bearing capacity of the components. For ordinary-strength steel, the residual stress has a very obvious influence on the overall and local stable bearing capacity of the axially compressed round tube short column, while for the high-strength steel round tube member with higher yield strength, its distribution mode has a greater influence on the stable bearing capacity of the high-strength steel.

5.3. Parametric Finite Element Modeling Analysis. Finite element modeling usually requires a lot of time for users, and the modeling process is cumbersome and error-prone. The secondary development option based on the Python programming language in ABAQUS provides great convenience for users' parametric finite element modeling. The secondary development of ABAQUS is mainly divided into two parts: subprogram development and user graphical interface program development. The development of core calculation subprograms is based on the Fortran language. Users can use the interface to write their own material constitutive models according to different needs and can create new unit types; the user graphical interface (GUI) program is a secondary development platform based on the Python language, and users can use Python language to write programs based on specific modeling needs. It can be expanded and can independently develop pre- and postprocessing modules and GUI tools to realize a series of parametric modeling processes.

The main research content of this subject is the local stability of high-strength steel tube axially compressed short columns. It is necessary to build more than 300 finite element short column models on the basis of verified finite element models. The number of models is large, the repeated 
operations are many, and the parameters are met. Therefore, in order to avoid errors in the manual modeling process and improve the efficiency of finite element analysis, this paper uses the Python language to write the corresponding script (Script) file and uses the ABAQUS environment to run the script to realize the batch creation, modification, and submission of the finite element model. The main content includes component generation, material attribute assignment, analysis step establishment, interaction definition, meshing, inp file modification, etc.

The parametric modeling process of the elastoplastic buckling problem of cylindrical short columns under axial compression is mainly divided into two steps, namely, the first-order linear elastic buckling modal analysis (buckle) and the second-order nonlinear elastoplastic buckling analysis. The key to buckling analysis lies in the application of geometric initial imperfections, as shown in Figure 2. In ABAQUS, the initial geometric defects cannot be introduced through the user graphical interface. Therefore, the key point to realize the parametric finite element analysis is to process the inp file in batches and add keywords in the specified line to introduce the initial geometric defects of the component.

\section{Structure and Calculation Model of High- Strength Steel Composite Local Buckling Fiber Hinged Compression Rod}

6.1. Flange Core-Casing High-Strength Steel Composite Local Buckling Fiber Hinge Structure. The buckling load of the axial compression member is determined by the restraint of the end, the calculated length factor, the section characteristics, and the material characteristics. In this section, the addition of a hoop-type outer tube device provides effective constraints on the hinged core steel tube at both ends to reduce the effective calculation length, thereby increasing the buckling load of the core steel tube. Figure 3 shows the theoretical buckling mode of the inner core steel pipe after adding constraints.

Flange-type core-sleeve combination local buckling fiber hinged rods include high-strength steel core steel pipes, ordinary steel outer sleeves, flange discs, connecting bolts, rubber ring blocks, and rubber gaskets. The number of outer casing pipes is generally 4 (if the slenderness ratio of the inner steel pipe is less than 80 , it can be reduced to 2 ; if the slenderness ratio of the inner steel pipe is greater than 150, it can be increased to 6). The two outer sleeves at the upper and lower ends are welded with flange discs at only one end, while the two outer sleeves in the middle are welded with flange discs at both ends; the inner diameter of the flange disc is the outer diameter of the core steel pipe, and the outer flanges of the sleeve bolt holes are arranged at equal intervals along the periphery of the disk. The inner core steel pipe is the main compression component, and a flange disc is welded in its middle part, and its outer diameter is the same as the outer diameter of the outer pipe flange. The inner core steel pipe and the outer sleeve and the outer sleeve are connected as a whole by a flange disc and high-strength bolts. Rubber round gaskets are added between each flange disc to play a sealing role. You fill rubber ring blocks at both ends of the outer tube and the corresponding positions of the inner core steel tube to seal and fix the inner core steel tube. There is a gap between the outer casing and the inner core steel tube, so that the weight of the composite component is lighter, and the inner core steel tube can freely undergo axial deformation, which plays a major role in resisting the axial pressure.

The outer tube 2 of ordinary strength grade steel does not participate in the force and only serves as a restraint. The number of outer tubes is generally 4 (if the core steel tube slenderness ratio is less than 80 , it can be reduced to 2 ; if the core steel tube slenderness ratio is greater than 150 , it can be increased to 6). The two outer sleeves at the upper and lower ends are welded with flange disc 3 only at one end, and the two outer sleeves at the middle are welded with flange disc 3 at both ends. The inner diameter of the flange disc 3 is the outer diameter of the core steel pipe, and four bolt holes are arranged at equal intervals along the periphery. The inner core steel pipe and the outer sleeve and the outer sleeve are connected as a whole through a flange disc and highstrength bolts. There is a gap between the outer casing and the inner core steel tube, so that the weight of the composite member is lighter, and the inner core steel tube can freely undergo axial deformation. Rubber round gaskets are added between each flange disc to play a sealing role.

6.2. Establishment of Finite Element Model of Inner Core Steel Pipe. The establishment of the correct finite element model of the inner core steel tube is a necessary prerequisite for the establishment of the finite element model of the composite local buckling fiber hinged rod. Therefore, in this section, five core steel pipe finite element models with slenderness ratios are established based on ANSYS software. The element adopts three-dimensional finite strain beam element BEAM188 (suitable for analyzing various beam-column members with large to medium slenderness ratios), and each member is divided into 20 elements (verified, the number of divided elements meets the accuracy requirements). The model created at this time has no geometric initial defects and residual stress.

The displacement constraint is shown in Figure 4. In the model, the $y$-axis passes through the center of the section along the length of the member. Since the circular section is a section with equal moment of inertia, the $x$-axis is artificially assumed to be the weak axis, so that the member undergoes first-order buckling around the $x$-axis. Therefore, the torsional deformation and $x$-direction translational displacement of all nodes in the model are restricted (otherwise, although static analysis can be carried out, abnormal buckling modes will occur), the $z$-direction displacement at both ends of the member and the $y$-direction displacement at the bottom end are also restricted.

6.3. Finite Element Model of Hoop-Type Composite Locally Buckled Fiber Hinged Compression Rod. In this paper, based on the construction method of the flange-type composite local buckling fiber hinged rod, the solid element (SOLID95) 

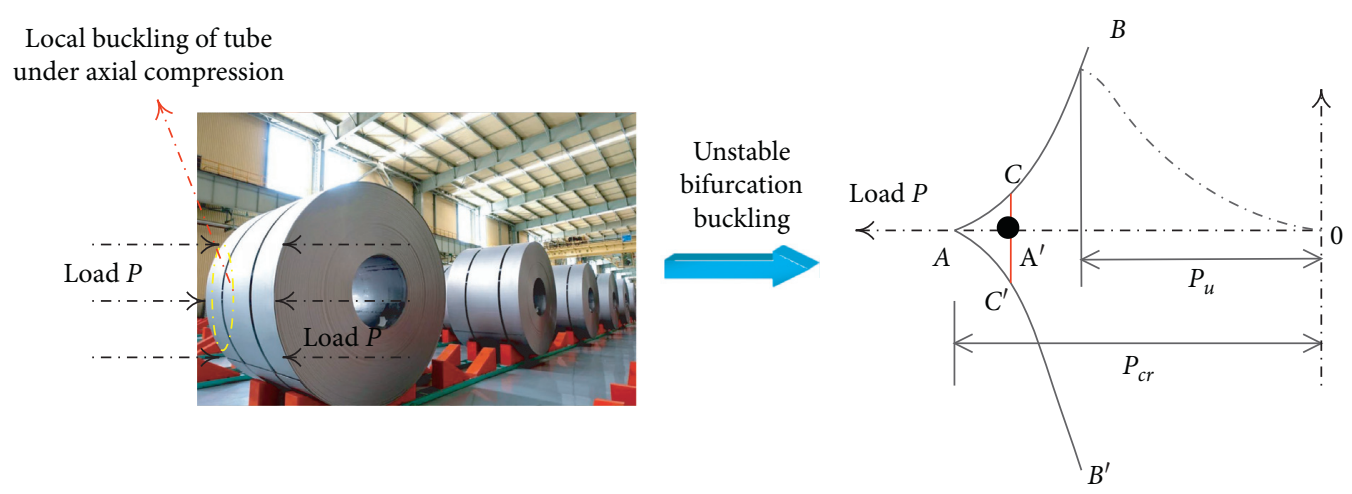

FIGURE 1: Local buckling and bifurcation instability of circular tube.

finite element model and the beam element (BEAM188) finite element model are established to analyze the eigenvalue buckling. Among them, the flange connection in the beam element model adopts the simplified processing of node coupling.

Both the core steel tube and the outer tube are simulated by three-dimensional finite strain beam elements BEAM188. The number of elements of the outer tube and the inner tube depends on the length of different components, ranging from 20 to 35 (to ensure that each finite element model has a relatively high accuracy).

The constitutive model of steel material in this paper adopts a multilinear follow-up strengthening model. The calculation process of finite element analysis is carried out in three steps. First, we create a finite element model of a ferrule-type composite locally buckled fiber hinged compression rod without initial defects and then perform elastic buckling analysis. The external load is continuously increased through cyclic iterations, and finally the buckling load factor is 1.0, thus obtaining the corresponding elastic buckling load and first-order instability mode. Finally, the elastoplastic stability bearing capacity analysis of the local buckling fiber hinged compression rod model of the highstrength steel composite is carried out. Based on the uniform defect model (that is, the first-order modal solution based on the eigenvalue buckling analysis), a peak value of $L / 1000$ is applied. Then the arc length method is used to solve the problem, and the minimum number of substeps required for the best effective solution is determined through continuous attempts to ensure the calculation accuracy and achieve the highest calculation efficiency.

\section{High-Strength Steel Nonlinear Detection Results and Analysis}

7.1. Data Collection and Processing. Properly we process the experimental signals obtained through the nonlinear ultrasonic system to obtain the magnitude of the fundamental wave and the second harmonic and then calculate the value of the ultrasonic nonlinear parameter. Ultrasound is expressed in two ways: time domain signal and frequency domain signal. The time domain signal represents the relationship between the magnitude of the ultrasonic amplitude and time. The frequency domain signal reflects the amplitude distribution of the ultrasonic wave on the frequency components.

The pulse signal is excited by the RAM-5000 high-energy ultrasound system, passes through an attenuator for $9 \mathrm{db}$ attenuation, enters a $0.4 \mathrm{MHz}$ low-pass filter for filtering, and then is transmitted to a $1.2 \mathrm{MHz}$ ultrasonic piezoelectric transducer. The device converts it into an ultrasonic signal and transmits it to the sample. The probe at the receiving end converts the received ultrasonic signal into an electrical signal. The receiving probe transmits the signal to $1.6 \mathrm{MHz}$ high-pass filtering and $20 \mathrm{db}$ preamplifier for processing and then to the oscilloscope and computer for data processing and analysis. What the computer extracts is the time domain signal, as shown in Figure 5. The signal collected by the computer can be processed by short-time Fourier transform (STFT) or fast Fourier transform (FFT) to obtain frequency domain signals. For the signal obtained in this experiment, because the signals of different modes of the received signal overlap each other in the time domain, the effect of fast Fourier transform is not good. Therefore, in this experiment, the short-time Fourier transform is used to process all the received signals.

7.2. Research on the Relationship between Nonlinear Parameters and Propagation Distance. The FV520 B highstrength steel original plate specimen that has not undergone the fatigue test is used as the experimental object to study the relationship between the ultrasonic nonlinear parameters and the propagation distance of the ultrasonic wave in the FV520B thin metal plate. We keep the incident voltage unchanged and change the distance between the transmitter probe and the receiver probe. The distance between the transmitter probe and the receiver probe is set to $40 \mathrm{~mm}$ to $80 \mathrm{~mm}$, and a test is performed every $10 \mathrm{~mm}$ increase.

The relationship between the ultrasonic nonlinear parameter $\beta$ and the distance that the ultrasonic wave propagates in the FV520B sample is shown in Figure 6. At this time, the tested sample is the original FV520B high-strength steel sample without fatigue test. The test results show that the nonlinear response can still be detected in the undamaged intact sample, indicating that the nonlinear response here is due to the inherent nature of the medium. It 

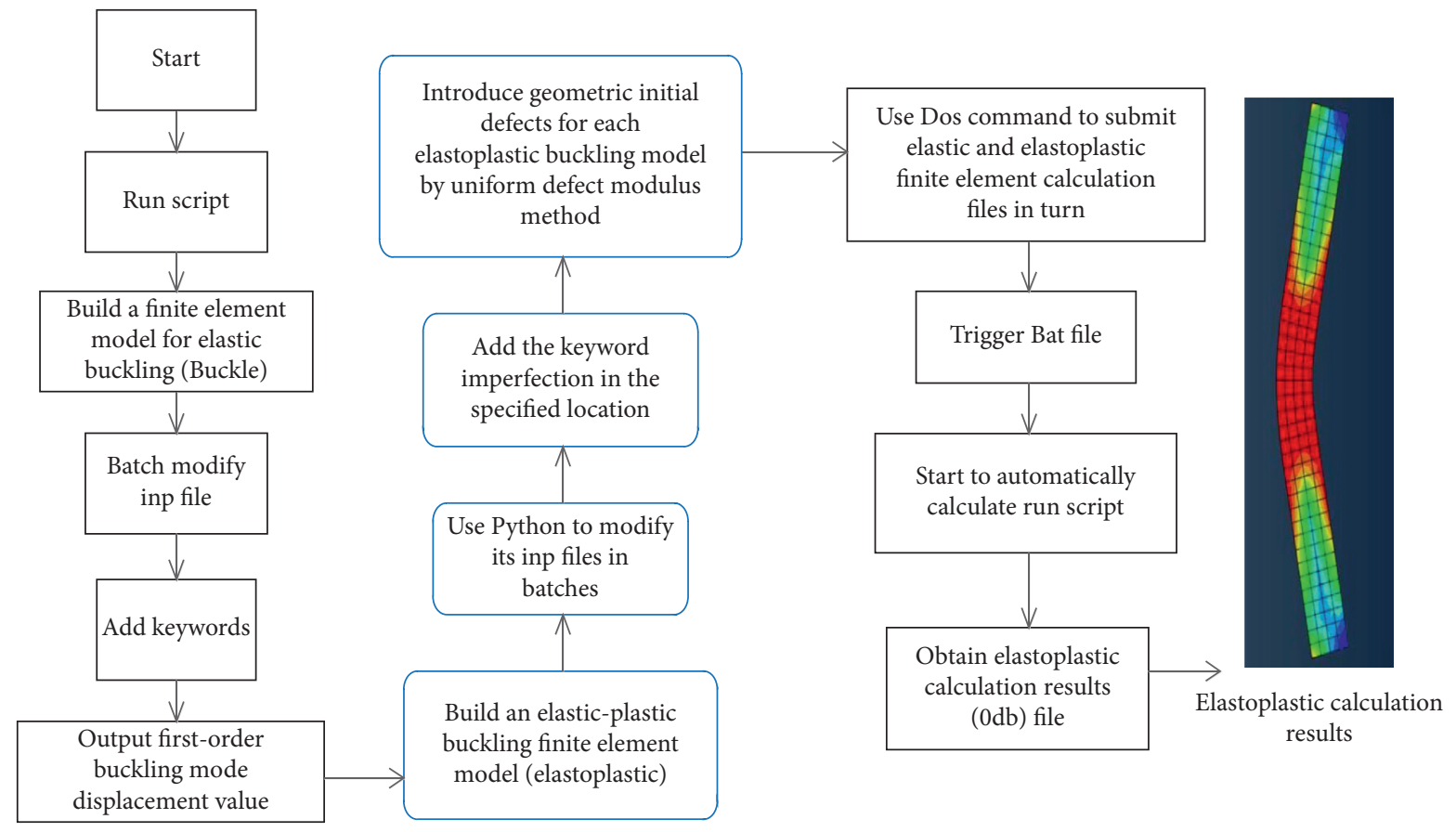

Figure 2: Parametric elastoplastic buckling analysis process.

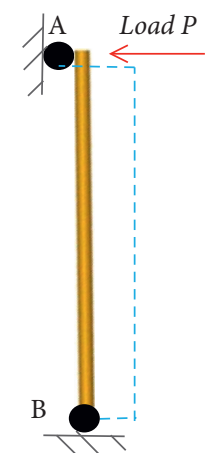

(a)

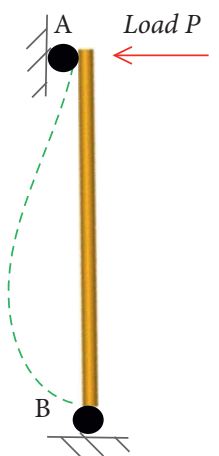

(b)

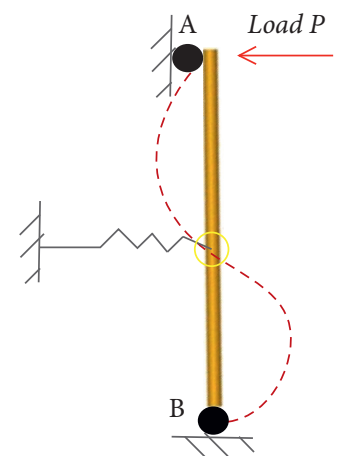

(c)

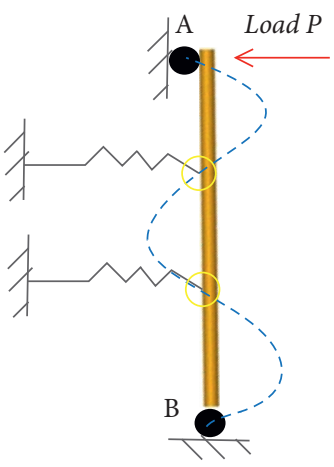

(d)

FIGURE 3: Schematic diagram of inner tube buckling.

can be considered that the internal microscopic features of the original sample are uniformly distributed, and the ultrasonic nonlinear response is caused by the nonharmonicity of the crystal lattice. The experimental results show that the ultrasonic nonlinear parameters increase approximately linearly as the propagation distance increases. The results show that as its propagation distance increases, the ultrasonic nonlinear response has a cumulative effect.

The relationship between the ultrasonic nonlinear parameter $\beta$ and the propagation distance of the ultrasonic wave in the FV520B sample is shown in Figure 7. It can be concluded from Figure 7 that as the propagation distance increases, the ultrasonic nonlinear parameters have a cumulative effect, and the results of the original test on the undamaged plate shape show that the nondamaged FV520B high-strength steel medium itself has nonlinearity.
According to the test results of notched specimens, it can be seen that the contribution of cracks to the ultrasonic nonlinear parameters is greater than the contribution of dislocations to the ultrasonic nonlinear parameters. The tested sample is a FV520B high-strength steel notched sample subjected to a certain fatigue cycle. After the fatigue test, cracks appear in the notch of the notched sample. Because the specimen is in a state of stress concentration at the notch, the stress it receives is much higher than the area far away from the notch. After the fatigue test, a large number of cracks appear near the notch, and no cracks are found in the area far away from the notch, which stays before the crack initiation. The dislocation stage is shown in Figure 8. This is consistent with the idea that the ultrasonic nonlinear response induced by cracks is greater than the ultrasonic nonlinear response induced by dislocations. 

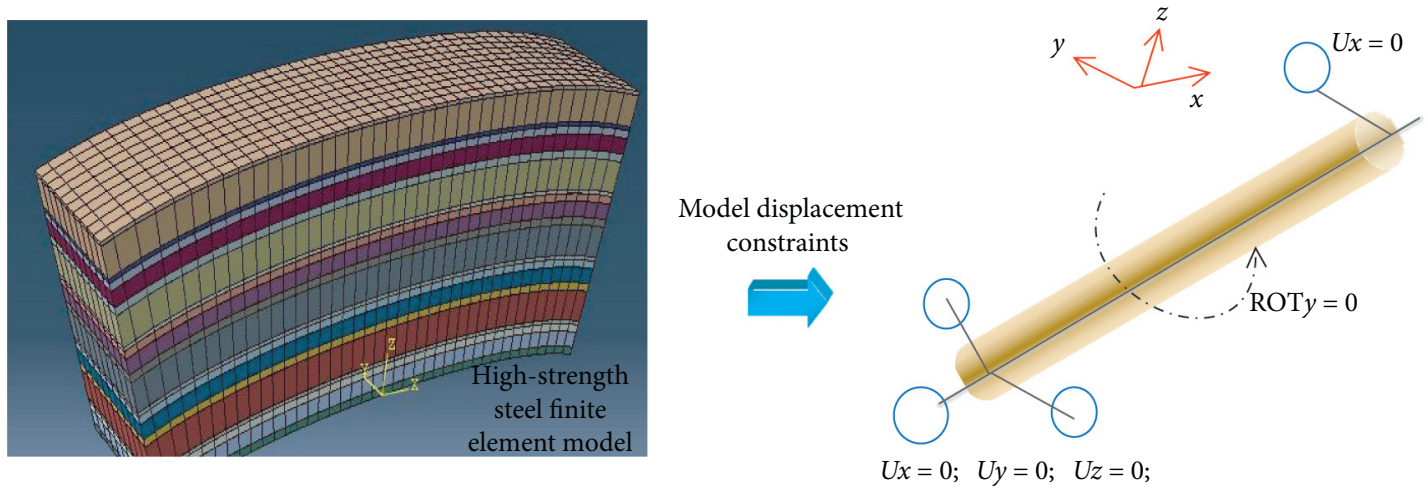

FIGURE 4: Schematic diagram of model displacement constraint.

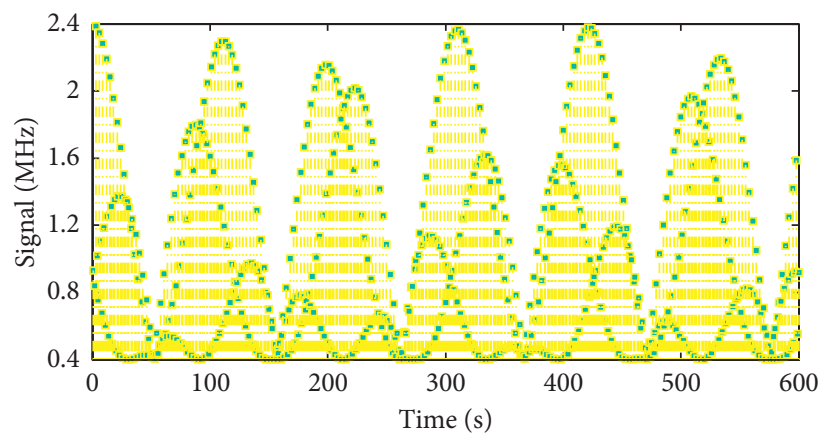

FIgURE 5: Time domain signal extracted by experiment.

\subsection{Research on the Relative Position of Nonlinear Parameters} and Cracks between Two Sensors. In this section, the notched sample after fatigue damage appears as the experimental object, and the influence of the relative position of the crack between the two ultrasonic transducers on the ultrasonic nonlinear parameters is studied. The distance between the transmitting probe and the receiving probe is always maintained at $60 \mathrm{~mm}$, and the distance between the transmitting probe and the crack center at the notch is $10 \mathrm{~mm}$, $20 \mathrm{~mm}, 30 \mathrm{~mm}, 40 \mathrm{~mm}$, and $50 \mathrm{~mm}$. Each time a group is tested, the transmitting probe and the receiving probe must move $10 \mathrm{~mm}$ at the same time to achieve the change of the relative position of the crack between the two probes.

Specimen No. 03 is a test piece of group A notch test (550 MPa) cycled for 105 cycles. The relationship between the ultrasonic nonlinear parameters of No. 03 specimen and the distance between the transmitting probe and the crack is shown in Figure 9. The test results show that the size of the ultrasonic nonlinear parameters will change with the position of the crack between the two sensors.

Specimen No. 04 is a specimen of group B notch experiment $(550 \mathrm{MPa})$ cycled for 105 cycles. The relationship between the ultrasonic nonlinear parameters of the specimen and the distance between the transmitting probe and the crack is shown in Figure 10. It can be seen from the figure that, unlike the test result of No. 03 specimen, the magnitude of the ultrasonic nonlinear parameter fluctuates greatly with the change of the position of the crack at the two ends between the two sensors.
Comparing the nonlinear ultrasonic detection results of the above two experiments, it can be concluded that the change of the position of the crack between the two sensors has a greater impact on the ultrasonic nonlinear parameters. Comparing Figure 9 and Figure 10, it can be found that the ultrasonic nonlinear parameter of No. 04 sample in group B is larger than the value of the ultrasonic nonlinear parameter of No. 03 sample in group A. This is because the fatigue cycle cycles of these two specimens are both $10^{5}$ times, and the loading stress $(660 \mathrm{MPa})$ of the No. 04 specimen in group B is greater than the loading stress of the No. 03 specimen in group A (550 MPa). This shows that the greater the loading stress of the fatigue experiment, the greater the value of the corresponding ultrasonic nonlinear parameter. There is a mapping relationship between the nonlinear coefficient and the degree of fatigue damage of FV520B high-strength steel.

7.4. Research on the Variation Law of Nonlinear Parameters with Fatigue Cycles. In order to study the change law of ultrasonic nonlinear parameters of fatigue specimens in different cycles of fatigue cycles, a group of plate-shaped specimens and two groups of fatigue specimens with different loading stresses are used as the research objects, and the different cycle cycles of each group are analyzed. The second fatigue specimen is tested. The relationship between normalized nonlinear parameters and fatigue cycle is used to describe the nonlinear changes of materials due to fatigue damage. 


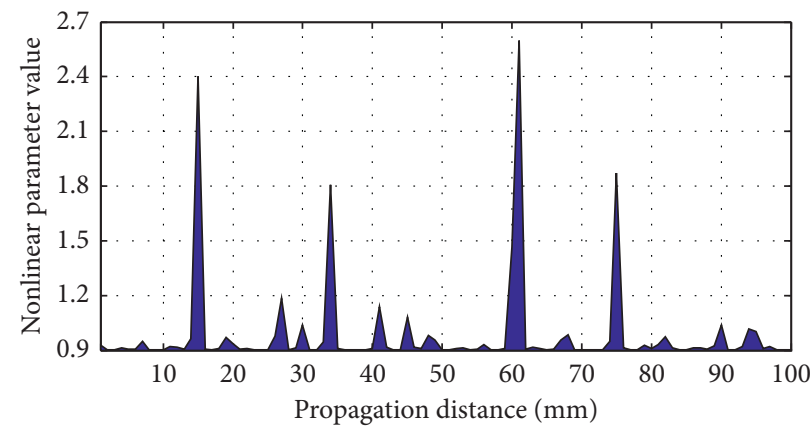

FIGURE 6: The relationship between ultrasonic nonlinear parameter $\beta$ and ultrasonic propagation distance.

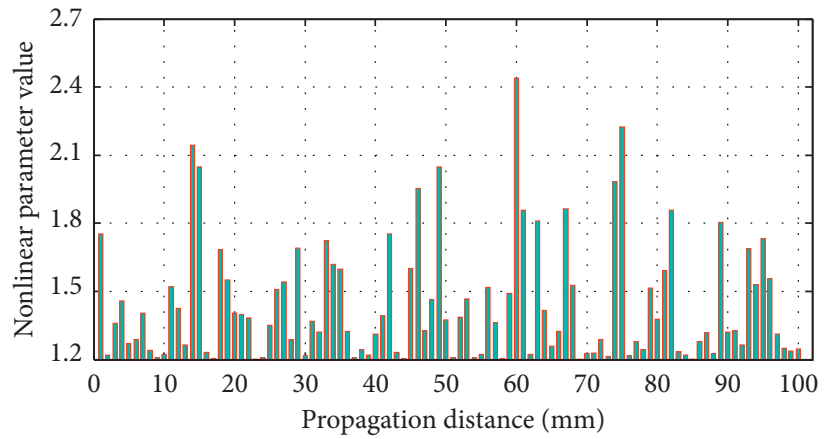

Figure 7: The relationship between the ultrasonic nonlinear coefficient $\beta$ and the distance between the transducer and the gap.

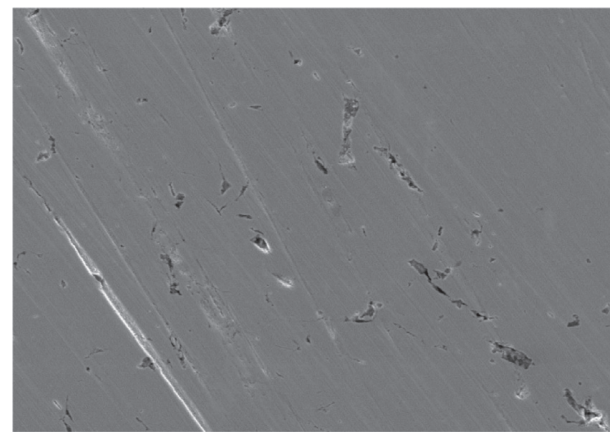

(a)

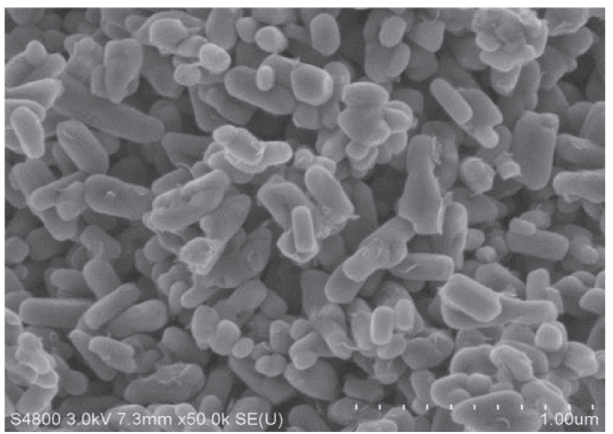

(b)

FIgURE 8: Electron micrograph of the cross section of the notched sample (a) away from the notched area and (b) the notched area.

In order to study the effect of fatigue crack growth on ultrasonic nonlinear parameters, we designed two sets of notched specimens. Figure 11 is the relationship curve between normalized ultrasonic nonlinear parameters and fatigue cycle cycles of the notched specimens in group A (550 MPa). Figure 12 shows the relationship between the normalized ultrasonic nonlinear coefficients of the notched specimens in group B (660 MPa) and fatigue cycle cycles. Ultrasonic nonlinear parameters are very sensitive to the fatigue damage of FV520B high-strength steel materials. The relationship between FV520B high-strength steel's nonlinear parameters and fatigue cycle cycles can be used to characterize its early fatigue.
By studying the relationship between ultrasonic nonlinear parameters and fatigue cycle cycles, it can be found that there is a good correlation between the ultrasonic nonlinear parameters of FV520B high-strength steel and fatigue cycle cycles. Nonlinear parameters can be used to reflect the fatigue life of materials. In engineering practice, after obtaining enough sample data points, the nonlinear parameter curve of the engineering component of FV520B high-strength steel material is calibrated in advance. Comparing the nonlinear detection results with the calibration curve obtained in advance, the fatigue life can be evaluated. 


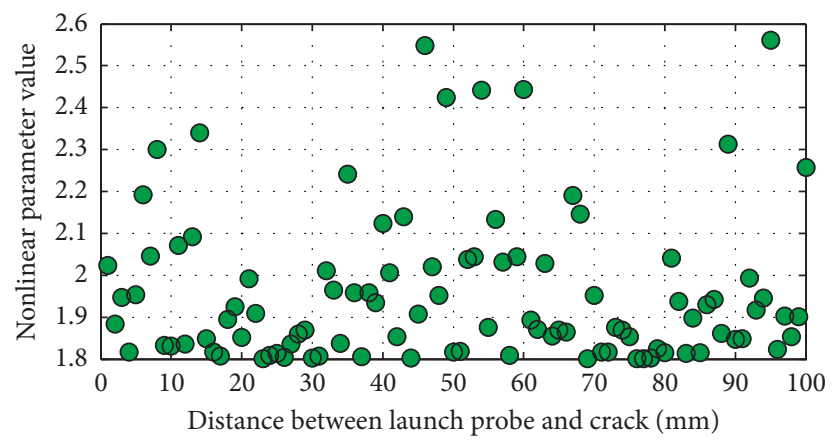

Figure 9: The relationship between the ultrasonic nonlinear parameters of No. 03 sample and the distance between the transmitting probe and the crack.

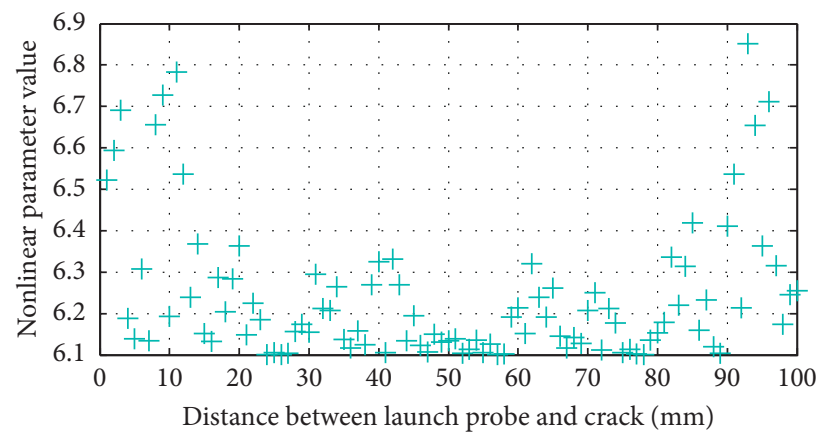

Figure 10: The relationship between the ultrasonic nonlinear parameters of No. 04 sample and the distance between the transmitting probe and the crack.

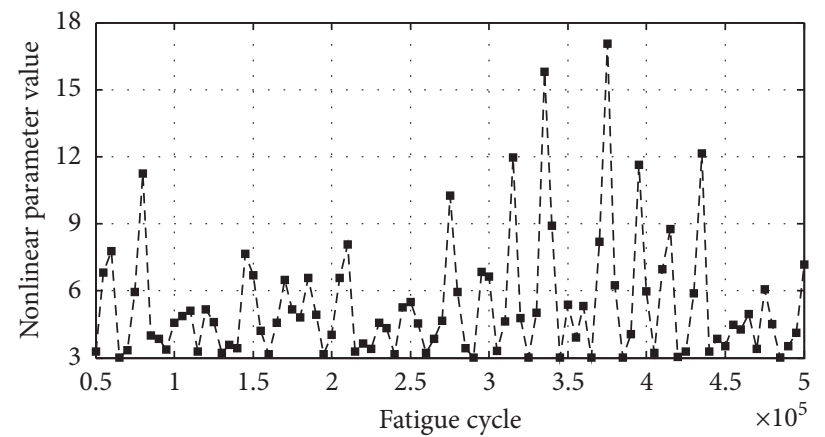

FIGURE 11: The relationship between normalized ultrasonic nonlinear parameters and fatigue cycle cycles for notched specimens (550 MPa).

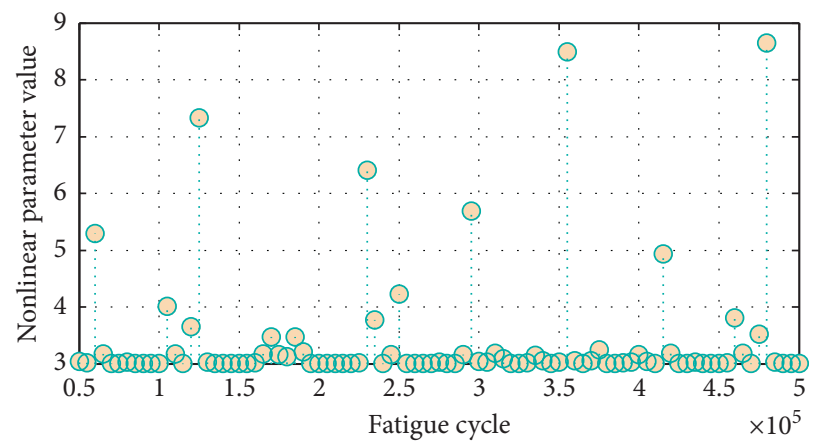

FIGURE 12: The relationship between normalized ultrasonic nonlinear parameters and fatigue cycle cycles for notched specimens (660 MPa). 


\section{Conclusion}

The outer steel tube combined with the local buckling fiber hinged compression rod has a good restraint effect on the inner core tube, and the bearing capacity of the combined local buckling fiber hinged compression rod increases with the increase of the outer and inner stiffness ratio and the number of hoops. However, an excessively large ratio of external and internal stiffness will cause the instability mode of the composite locally buckled fiber hinged rod to change from common instability to the inner core alone, which will reduce the bearing capacity of the composite locally buckled fiber hinged rod. For the core tube of high-strength and ultra-high-strength steel, the stable bearing capacity of the composite local buckling fiber hinged compression rod becomes more obvious with the increase of the slenderness ratio of the core tube. The influence of related parameters in the dislocation string model and the dislocation couple model on the ultrasonic nonlinear parameters is analyzed. From the perspective of contact nonlinear acoustics, the influence of cracks on ultrasonic nonlinearity is analyzed, and the finite element software ABAQUS is used to simulate it. It is proved that the crack can produce nonlinear effects, and the relationship between nonlinear parameters and the internal crack shape of the material is analyzed. The effect of the relative position of the crack between the two transducers on the ultrasonic nonlinear coefficient is studied, and it can be concluded that the change of the position of the crack between the two transducers has little effect on the nonlinear coefficient, which can be approximated as no effect. By comparing two fatigue specimens with the same fatigue cycle but different fatigue loading stress, it is found that the larger the fatigue test loading stress, the larger the corresponding ultrasonic nonlinear parameter value. There is a mapping relationship between the nonlinear coefficient and the degree of fatigue damage. Lamb waves are used to perform a series of nonlinear tests on fatigue specimens of FV520B high-strength steel. Analyzing the results, it is found that the material has a good ultrasonic nonlinear cumulative effect. The experimental results show that the ultrasonic nonlinear parameters have high sensitivity to the early fatigue damage of FV520 B high-strength steel.

\section{Data Availability}

Data sharing is not applicable to this article as no datasets were generated or analyzed during the current study.

\section{Consent}

Informed consent was obtained from all individual participants included in the study references.

\section{Conflicts of Interest}

The authors declare that there are no conflicts of interest.

\section{Acknowledgments}

This work was supported by the Natural Science Foundation of the Jiangsu Higher Education Institutions of China (No.
19KJB560018) and by the Jiangsu Province Vocational Colleges Young Teacher Enterprise Practice Training Project (No. 2020QYSJ165).

\section{References}

[1] D. Wu, Z. Liu, X. Wang, and L. Su, "Composite magnetic flux leakage detection method for pipelines using alternating magnetic field excitation," NDT \& E International, vol. 91, pp. 148-155, 2017.

[2] J. Abrahamsson, M. Hedlund, T. Kamf, and H. Bernhoff, "High-speed kinetic energy buffer: optimization of composite shell and magnetic bearings," IEEE Transactions on Industrial Electronics, vol. 61, no. 6, pp. 3012-3021, 2014.

[3] P. J. Janse Van Rensburg, A. A. Groenwold, and D. W. Wood, "Optimization of cylindrical composite flywheel rotors for energy storage," Structural and Multidisciplinary Optimization, vol. 47, no. 1, pp. 135-147, 2013.

[4] K. M. Lee, S. Y. Park, M. Y. Huh, J. S. Kim, and O. Engler, "Effect of texture and grain size on magnetic flux density and core loss in non-oriented electrical steel containing 3.15\% Si," Journal of Magnetism and Magnetic Materials, vol. 354, pp. 324-332, 2014.

[5] P. B. Reddy, A. M. El-Refaie, S. Galioto, and J. P. Alexander, "Design of synchronous reluctance motor utilizing dual-phase material for traction applications," IEEE Transactions on Industry Applications, vol. 53, no. 3, pp. 1948-1957, 2017.

[6] Y. Zhong, L.-E. Rännar, L. Liu et al., "Additive manufacturing of 316L stainless steel by electron beam melting for nuclear fusion applications," Journal of Nuclear Materials, vol. 486, pp. 234-245, 2017.

[7] Y. Gao, G. Y. Tian, K. Li, J. Ji, P. Wang, and H. Wang, "Multiple cracks detection and visualization using magnetic flux leakage and eddy current pulsed thermography," Sensors and Actuators A: Physical, vol. 234, pp. 269-281, 2015.

[8] D. S. Petrovic, B. Markoli, and M. Ceh, "The nanostructure of non-oriented electrical steel sheets," Journal of Magnetism and Magnetic Materials, vol. 322, no. 20, pp. 3041-3048, 2010.

[9] S. Ghanei, M. Kashefi, and M. Mazinani, "Comparative study of eddy current and Barkhausen noise nondestructive testing methods in microstructural examination of ferrite-martensite dual-phase steel," Journal of Magnetism and Magnetic Materials, vol. 356, pp. 103-110, 2014.

[10] I. Tanaka, H. Nitomi, K. Imanishi, K. Okamura, and H. Yashiki, "Application of high-strength nonoriented electrical steel to interior permanent magnet synchronous motor," IEEE Transactions on Magnetics, vol. 49, no. 6, pp. 2997-3001, 2013.

[11] S. Şahin and M. Übeyli, "A review on the potential use of austenitic stainless steels in nuclear fusion reactors," Journal of Fusion Energy, vol. 27, no. 4, pp. 271-277, 2008.

[12] G. Yang, Z. Zeng, Y. Deng et al., “3D EC-GMR sensor system for detection of subsurface defects at steel fastener sites," NDT \& E International, vol. 50, pp. 20-28, 2012.

[13] Y. Chang, J. Jiao, G. Li, X. Liu, C. He, and B. Wu, "Effects of excitation system on the performance of magnetic-fluxleakage-type non-destructive testing," Sensors and Actuators A: Physical, vol. 268, pp. 201-212, 2017.

[14] J. Gong and H. W. Luo, "Progress on the research of highstrength non-oriented silicon steel sheets in traction motors of hybrid/electrical vehicles," Journal of Materials Engineering and Performance, vol. 43, no. 6, pp. 102-112, 2015.

[15] S. Liu, "A new signal processing method based on notch filtering and wavelet denoising in wire rope inspection," 
Journal of Nondestructive Evaluation, vol. 38, no. 2, pp. 1-14, 2019.

[16] P. Libeyre, N. Mitchell, D. Bessette, Y. Gribov, C. Jong, and C. Lyraud, "Detailed design of the ITER central solenoid," Fusion Engineering and Design, vol. 84, no. 7-11, pp. 11881191, 2009.

[17] P. Zheng and J. Zhang, "Quantitative nondestructive testing of wire rope based on pseudo-color image enhancement technology," Nondestructive Testing and Evaluation, vol. 34, no. 3, pp. 221-242, 2019.

[18] Z. Lv, P. Cai, T. Yu et al., "Fatigue behaviors and damage mechanism of a Cr-Mn-N austenitic steel," Journal of Alloys and Compounds, vol. 691, pp. 103-109, 2017.

[19] M. Zhao, D. Zhang, and Z. Zhou, "The research on quantitative inspection technology to wire rope defect based on Hall sensor array," Nondestructive Testing, vol. 34, no. 11, pp. 57-60, 2012.

[20] D. L. Zhang, Y. N. Cao, C. Wang, and D. G. Xu, "A new method of defects identification for wire rope based on threedimensional magnetic flux leakage," Journal of Physics: Conference Series, vol. 48, no. 1, pp. 334-338, 2006.

[21] H. Ozeki, K. Hamada, Y. Takahashi et al., "Establishment of production process of JK2LB jacket section for ITER CS," IEEE Transactions on Applied Superconductivity, vol. 24, no. 3, pp. 1-4, 2014.

[22] E. Arias-Castro and D. L. Donoho, "Does median filtering truly preserve edges better than linear filtering?" Annals of Statistics, vol. 37, no. 3, pp. 1172-1206, 2009.

[23] A. Nyilas, "Fatigue crack growth rate and fracture toughness of ITER central solenoid jacket materials at $7 \mathrm{~K}$," AIP Conference Proceedings, vol. 1435, no. 1, pp. 47-54, 2012. 